ARTICLE

\title{
Catalyst-free carbosilylation of alkenes using silyl boronates and organic fluorides via selective $\mathrm{C}-\mathrm{F}$ bond activation
}

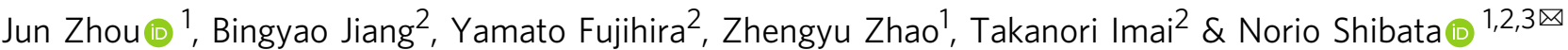

\begin{abstract}
A regioselective carbosilylation of alkenes has emerged as a powerful strategy to access molecules with functionalized silylated alkanes, by incorporating silyl and carbon groups across an alkene double bond. However, to the best of our knowledge, organic fluorides have never been used in this protocol. Here we disclose the catalyst-free carbosilylation of alkenes using silyl boronates and organic fluorides mediated by ${ }^{t} \mathrm{BuOK}$. The main feature of this transformation is the selective activation of the $\mathrm{C}-\mathrm{F}$ bond of an organic fluoride by the silyl boronate without undergoing potential side-reactions involving $\mathrm{C}-\mathrm{O}, \mathrm{C}-\mathrm{Cl}$, heteroaryl- $\mathrm{CH}$, and even $\mathrm{CF}_{3}$ groups. Various silylated alkanes with tertiary or quaternary carbon centers that have aromatic, hetero-aromatic, and/or aliphatic groups at the $\beta$-position are synthesized in a single step from substituted or non-substituted aryl alkenes. An intramolecular variant of this carbosilylation is also achieved via the reaction of a fluoroarene with a $\omega$-alkenyl side chain and a silyl boronate.
\end{abstract}

\footnotetext{
${ }^{1}$ Department of Nanopharmaceutical Sciences, Nagoya Institute of Technology, Gokiso, Showa-ku, Nagoya, Japan. ${ }^{2}$ Department of Life Science and Applied Chemistry, Nagoya Institute of Technology, Gokiso, Showa-ku, Nagoya, Japan. ${ }^{3}$ Institute of Advanced Fluorine-Containing Materials, Zhejiang Normal

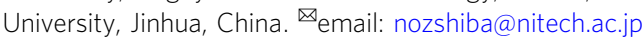


T he use of two independent reactants for the transitionmetal-catalyzed difunctionalization of alkenes has emerged as a powerful strategy to access molecules with multiple functional groups, by incorporating two new functional groups across an alkene double bond ${ }^{1-6}$. One of the two coupling partners commonly used in this context are activated halogenated arenes or alkanes, or an equivalent thereof (Ar-X or Alkyl-X; $X=$ e.g., I, $\mathrm{Br}, \mathrm{Cl}$, OTf, or OMs). Reactions using these molecules generally proceed via oxidative addition and/or radical processes ${ }^{7-15}$. In contrast, fluorinated compounds such as aryl or alkyl fluorides have not yet been used as a counterpart for the difunctionalization of alkenes due to the high bond-dissociation energy of the carbon-fluorine (C-F) bond (Fig. 1a).

In recent years, a variety of organofluorine compounds has become ubiquitous due to the rapid establishment of technologies for their synthesis ${ }^{16-18}$. There are more than 340 registered fluoro-pharmaceuticals ${ }^{19}$ with complex structures and more than 425 registered fluoro-agrochemicals ${ }^{20}$. It has been estimated that there are more than 8,800,000 commercially available fluoroarenes (SciFinder $\left.{ }^{\circledast}\right)$. Thus, the functionalization of organic fluorides via the cleavage/activation of $\mathrm{C}-\mathrm{F}$ bonds in organic fluorides is a dynamic and emerging area of research that will expand the utility of organofluorine molecules ${ }^{21-24}$. However, the chemical transformation of fluoro-organic compounds via $\mathrm{C}-\mathrm{F}$ bond cleavage under very mild conditions is still rare. For example, aryl fluorides have rarely been functionalized due to the difficulty of oxidative addition of low-valent transition metals to $\mathrm{C}\left(s p^{2}\right)-\mathrm{F}$ bonds, whereas the field of $\mathrm{C}\left(s p^{2}\right)$-F cleavage using silyl cooperativity and/or transition-metal catalysis has also benefited from a wide breadth of research, especially in the last decade ${ }^{24-30}$. Activation of $\mathrm{C}\left(s p^{3}\right)$-F bonds in aliphatic fluorocarbons is mostly limited to reactive benzyl or allylic fluorides where $p$-block-based Lewis acids with high fluoride affinity are usually required ${ }^{31-33}$. Thus, the activation of inert C-F bonds in aromatic and aliphatic organic fluorides under mild conditions remains highly challenging.

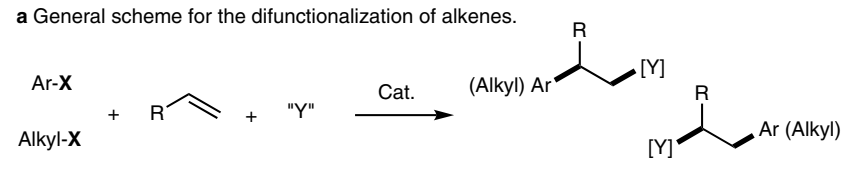

$\mathbf{X}=\mathrm{I}, \mathrm{Br}, \mathrm{Cl}, \mathrm{OTf}, \mathrm{OMs}$, etc., but not $\mathbf{F}$

b Carbosilylation of alkenes with silyl boronates and organic fluorides via C-F bond activation (this work).

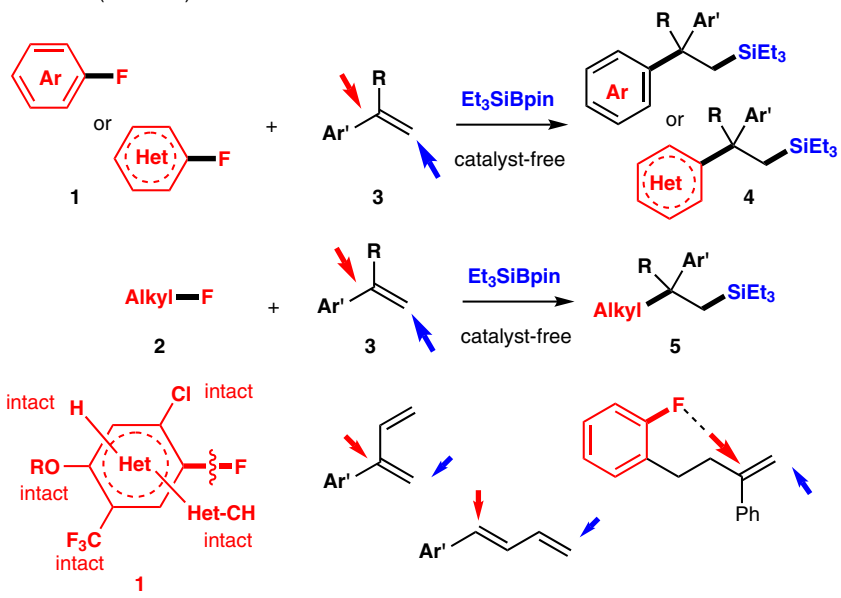

Fig. 1 Difunctionalization of alkenes. a General figure for the difunctionalization of alkenes. $\mathbf{b}$ Carbosilylation of alkenes with silyl boronates and organic fluorides.
To realize the difunctionalization of alkenes using organic fluorides as one of the coupling partners, we decided to investigate the carbosilylation of alkenes ${ }^{34-49}$. This reaction provides functionalized organosilicon compounds directly from simple alkenes, compounds that are much sought-after materials in organic synthesis ${ }^{50,51}$, electronics ${ }^{52,53}$, photonics ${ }^{54}$, and drug discovery 55 . Despite previous successes in the hydrosilylation of alkenes $^{56,57}$, research on the carbosilylation of alkenes is still in its infancy ${ }^{34-49}$. One of the early milestones in this area is the work by Terao and colleagues ${ }^{37}$. They disclosed a titanocene-catalyzed carbosilylation of alkenes using alkyl bromides and chlorides with chlorosilanes mediated by the Grignard reagent. For recent examples, Engle and colleagues ${ }^{42}$ reported a Pd-catalyzed arylsilylation of alkenes using dimethylphenyl silyl boronate $\mathrm{PhMe}_{2}-$ SiBPin and aryl triflates. Zhang and $\mathrm{Hu}^{49}$ demonstrated an arylsilylation of acrylates with tri(trimethylsilyl)silane and aryl bromides using a nickel/photoredox catalyst. Although several achievements have been reported in carbosilylation reactions of alkenes under metal-catalyzed and photo-induced conditions, the present methods are still limited by the substrate scope. Besides, the aryl and alkyl fluorides have never been used for the threecomponent carbosilylation of alkenes. Our group has been engaged in the development of efficient methods for the selective functionalization of $\mathrm{C}\left(s p^{2}\right)-\mathrm{F}$ and $\mathrm{C}\left(s p^{3}\right)$-F bonds under mild conditions both with ${ }^{58}$ or without ${ }^{58-61}$ the use of transition metals. In this context, our recent report on the defluorosilylation of organic fluorides using silyl boronates and with or without a $\mathrm{Ni}$ catalyst ${ }^{58}$ inspired us to undertake a much more challenging research topic: the difunctionalization of alkenes with aryl or alkyl fluorides in the presence of silyl boronates via $\mathrm{C}-\mathrm{F}$ bond cleavage (Fig. 1b).

Herein, we disclose a protocol consisting of the catalyst-free defluorinative carbosilylation of alkenes with silyl boronates and fluorinated compounds with an inert $\mathrm{C}\left(s p^{3}\right)-\mathrm{F}$ or $\mathrm{C}\left(s p^{2}\right)$-F bond. A wide variety of aryl fluorides and alkyl fluorides are smoothly incorporated into the alkenes via the cleavage of a C-F bond in the presence of silyl boronates to provide $\beta$-functionalized silyl alkanes in good to excellent yield, without any help of transitionmetal catalysis nor photoredox system. The alkene, aryl fluoride, and alkyl fluoride substrate scope tolerated by this reaction is extensive. Unsubstituted styrene derivatives as well as substituted and conjugated aryl alkenes react smoothly with a great variety of aryl or alkyl fluorides and silyl boronates. This allows access to a library of silyl compounds that regioselectively incorporate aryl, heteroaryl, and/or alkyl units at the $\beta$-carbon of the silyl alkanes. The reaction proceeds with high regio- and chemoselectivity. Aryl and conjugated alkenes are reactive, whereas non-aryl alkenes are entirely unreactive. The potentially cleavable $\mathrm{C}-\mathrm{O}$ bond of ethers $^{62}, \mathrm{C}-\mathrm{Cl}$ bonds 63 , and the $\mathrm{C}\left(s p^{2}\right)-\mathrm{H}$ bond of heteroaromatic compounds $s^{64,65}$ are well-tolerated. Most significantly, the $\mathrm{C}\left(s p^{3}\right)-\mathrm{F}$ bond of the trifluoromethyl $\left(\mathrm{CF}_{3}\right)$ group ${ }^{66}$ remains intact. An intramolecular carbosilylation via the cleavage and coupling of a C-F bond was also achieved. Three-component coupling reactions involving drug derivatives that contain a fluoride moiety were also demonstrated to prove the utility of this transformation in the drug discovery process. The reaction should proceed through a cascade radical process initiated by singleelectron transfer, which was supported by the experimental studies.

\section{Results and discussion}

Optimization of the reaction conditions. We first investigated the reaction of 4-fluorobiphenyl (1a) with styrene (3a) in the presence of the silyl boronate $\mathrm{Et}_{3} \mathrm{SiBpin}$ and a variety of catalysts (Table 1, also see Supplementary Tables S1-S5 for more details). 
Table 1 Optimization of the reaction conditions. ${ }^{a}$.

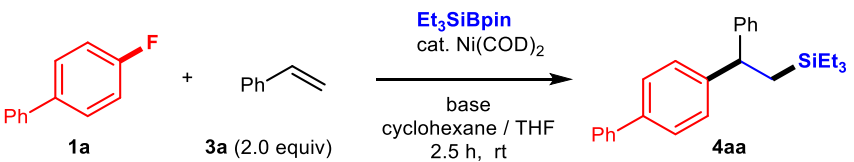

\begin{tabular}{|c|c|c|c|c|c|}
\hline Entry & $\mathrm{Ni}(\mathrm{COD})_{2}(\mathrm{~mol} \%)$ & $\mathrm{Et}_{3}$ SiBpin & Base (equiv) & Cyclohexane/THF & Yield $(\%)^{b}$ \\
\hline 1 & 10 & 1.5 & $\mathrm{KOtBu}(2.5)$ & $1 / 2$ & 32 \\
\hline 2 & 10 & 1.5 & KOtBu (3.5) & $1 / 2$ & 38 \\
\hline 3 & 10 & 1.5 & KOtBu (4) & $1 / 2$ & 52 \\
\hline 4 & 10 & 2 & KOtBu (4) & $1 / 2$ & 81 \\
\hline 5 & 10 & 2 & KOtBu (4) & $8 / 1$ & $98(95)$ \\
\hline 6 & 1 & 2 & $\mathrm{KOtBu}(4)$ & $8 / 1$ & $99(94)$ \\
\hline 7 & 1 & 2 & $\mathrm{NaOtBu}(4)$ & $8 / 1$ & 15 \\
\hline 8 & 1 & 2 & 0 & $8 / 1$ & 0 \\
\hline 9 & 1 & 2 & LiOtBu, KOMe or KHMDS (4) & $8 / 1$ & 0 \\
\hline $10^{c}$ & 1 & 2 & KOtBu (4) & $8 / 1$ & 45 \\
\hline 11 & 0 & 2 & $\mathrm{KOtBu}(4)$ & $8 / 1$ & 94 (91) \\
\hline
\end{tabular}

Using the conditions described in our earlier report ${ }^{58}$ on the defluorosilylation of aryl fluorides $\left[\mathrm{Et}_{3} \mathrm{SiBpin}\right.$ (1.5 equiv), $\mathrm{Ni}$ $(\mathrm{COD})_{2}(10 \mathrm{~mol} \%)$, and potassium tert-butoxide (KOtBu, 2.5 equiv) in cyclohexane/tetrahydrofuran (THF) at room temperature], the expected biphenyl-phenylethyl-triethylsilane 4aa was obtained regioselectively in $32 \%$ yield (entry 1 , Table 1). Encouraged by this initial attempt, the optimum amount of base required was explored and 4.0 equiv of $\mathrm{KO} t \mathrm{Bu}$ was found to be the most suitable quantity (entries $1-3$ ). We next varied the equivalents of $\mathrm{Et}_{3} \mathrm{SiBpin}$ used and found that 2.0 equiv was the optimum amount (entry 4). After slight modifications to the solvent ratios, including testing a single solvent, the binary solvent cyclohexane/THF $(8 / 1, v / v)$ was found to be most suitable (entry 5). Notably, reducing the $\mathrm{Ni}(\mathrm{COD})_{2}$ catalyst loading to 1 mol\% afforded 4 aa in $99 \%$ yield, which is similar to that obtained in entry 5 , within $2.5 \mathrm{~h}$ without eroding the reaction efficiency (entry 6). Replacement of $\mathrm{KO} t \mathrm{Bu}$ with $\mathrm{NaO} t \mathrm{Bu}$ resulted in the formation of 4aa in merely $15 \%$ yield (entry 7 ). In the absence of a base or when using other bases ( $\mathrm{LiO} t \mathrm{Bu}$, KOMe, or KHMDS), the desired product was not obtained (entries 8 and 9). Moreover, a large excess of $\mathbf{3 a}$ had a negative effect on the reaction yield (entry 10). To ascertain the effect of $\mathrm{Ni}(\mathrm{COD})_{2}$, we finally examined the reaction without $\mathrm{Ni}$ catalyst. We were very supersized that the transformation occurred efficiently even in the absence of $\mathrm{Ni}(\mathrm{COD})_{2}$ to generate $4 \mathbf{a a}$ in $94 \%$ yield (91\% isolated yield, entry 11). The results are almost identical to the reaction with $\mathrm{Ni}$ catalysis (entry 6). We thus decided the further examination using the different substrates under the two type conditions, with or without $\mathrm{Ni}(\mathrm{COD})_{2}$.

Substrate scope. With the optimized reaction conditions (Table 1, entries 6 and 11), we investigated the scope of the Ni-catalyzed or catalyst-free carbosilylation of the alkenes 3 with fluoroarenes 1 and $\mathrm{Et}_{3} \mathrm{SiBpin}$ (Fig. 2). First, the scope of the aromatic fluorides was examined under catalyst-free conditions (entry 11, Table 1). A wide range of fluoroarenes that bear $\pi$-extended building blocks were efficiently converted into the corresponding defluorinative arylsilylation products (4) in good to high yield. For example, biphenyl products (4aa: 91\%; 4ba: 87\%; 4ca: 42\%), a 1-naphthyl product (4da: $87 \%$ ), and 4-(naphthalen-1-yl)phenyl (4ea: $90 \%$ ) were all obtained using this methodology. Simple fluorobenzene (1f) was also efficiently converted into arylsilylation product $\mathbf{4 f a}$ (63\%), whereas 4-methyl-substituted aryl fluoride $\mathbf{1 g}$ provided a higher yield (4ga: $85 \%$ ). The excellent chemoselectivity profile of this process is nicely illustrated by the tolerance of the reaction conditions toward functional groups such as ethers (-O-: $\mathbf{1 h}, \mathbf{1 i}$, and $\mathbf{1 j}$ ), $\mathrm{CF}_{3}$ groups (1k and $\mathbf{1 l}$ ), or $\mathrm{Cl}$ groups (1m), all of which could potentially be cleaved with the C-F bond activation. The desired products 4 were obtained in the following yields: 4 ha: $75 \%$, 4ia: 73\%, 4ja: 69\%, 4ka: 50\%, 4la: 53\%, 4ma: 70\%. It was most surprising to find that the $\mathrm{CF}_{3}$ groups of $\mathbf{1 k}$ and $\mathbf{1 1}$ remained intact. We next repeated the same substrate scope by the best reaction conditions in the presence of $\mathrm{Ni}(\mathrm{COD})_{2}$ (entry 6, Table 1). Although the yield products 4aa-4na were somewhat improved, the differences were not so significant, as shown in Fig. 2.

Encouraged by the fact that the transformation fundamentally does not require Ni catalyst, we continued the substrate scope under catalyst-free conditions (entry 11, Table 1). Next, we focused on the hetero-aromatic fluorides $1 \mathbf{n}-\mathbf{1 u}$. The nitrogencontaining hetero-aromatic fluorides $\mathbf{1 n - 1 t}$ were successfully coupled with $\mathbf{3 a}$ in the presence of $\mathrm{Et}_{3} \mathrm{SiBpin}$ in good to high yield; a $1 H$-indole derivative (4na: 57\%), $1 H$-pyrrole derivative (4oa: 32\%), and a number of pyridine derivatives (4pa: $80 \%$; 4qa: 68\%; 4ra: 74\%; 4sa: $88 \%$ ) were well-tolerated under the optimized conditions. Fluoro-indole (1t) and fluoro-benzofuran (1u), despite having several reactive aryl $\mathrm{C}\left(s p^{2}\right)-\mathrm{H}$ bonds, also participated well in this transformation, selectively furnishing carbosilylation products 4 ta $(85 \%)$ and 4 ua (49\%) via C-F bond cleavage, without the anticipated $\mathrm{C}-\mathrm{H}$ activation-silylation reaction of the hetero-aromatic moiety. Notably, other silyl boronates such as $\mathrm{PhMe}_{2} \mathrm{SiBpin},{ }^{n} \mathrm{Pr}_{3} \mathrm{SiBPin}$, and ${ }^{t} \mathrm{BuMe}_{2} \mathrm{SiBPin}$, could also be used in this transformation instead of $\mathrm{Et}_{3} \mathrm{SiBpin}$. The corresponding silylated products 4aa', 4aa”, and 4aa"' were obtained from fluoroarene $1 \mathrm{a}$ and styrene (3a) in $47 \%, 88 \%$, and $94 \%$ yields, respectively.

Next, we set out to evaluate the scope of the alkene component (3) of the reaction. A range of aryl alkenes were efficiently converted into the corresponding arylsilylation products 4 in the presence of aryl fluoride $\mathbf{1}$ and $\mathrm{Et}_{3} \mathrm{SiBpin}$. 2-Vinylnaphthalene $\mathbf{3 b}$, a simple polycyclic aromatic compound, successfully reacted with 1a to afford the corresponding arylsilylation product (4ab, 58\%). Styrene derivatives bearing $\mathrm{Me}(\mathbf{3 c}),{ }^{t} \mathrm{Bu}(\mathbf{3 d})$, phenyl (3e), $\mathrm{MeO}$ 


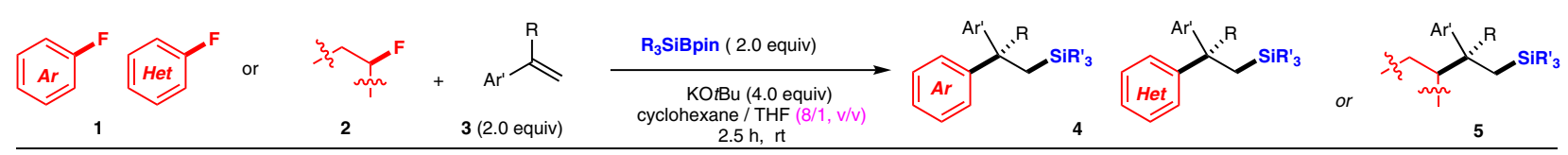

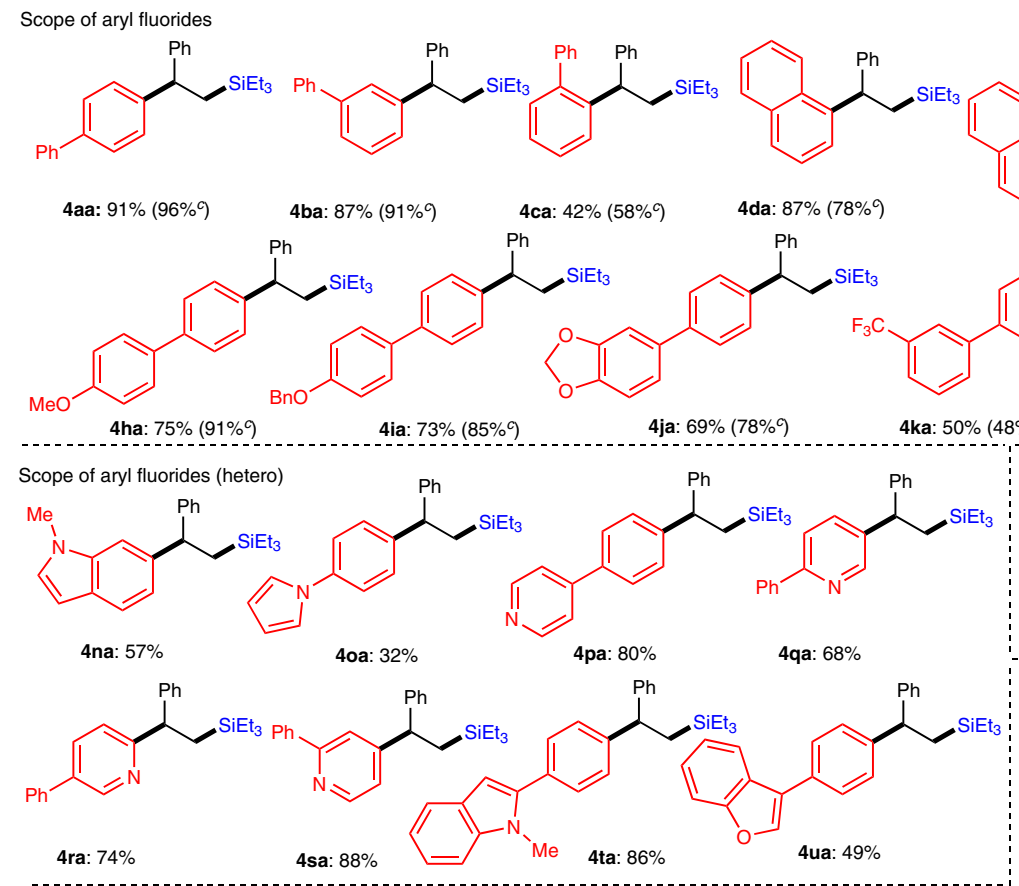

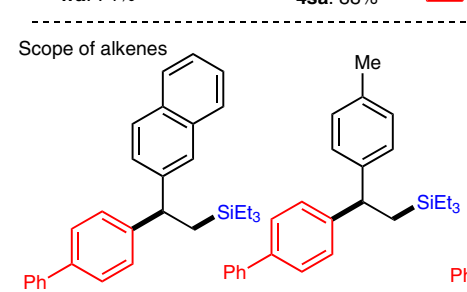<smiles>CCOCC(c1ccc(Br)cc1)c1ccc(-c2ccccc2)cc1</smiles><smiles>CCCC(c1ccc(I)cc1)c1ccc(-c2ccccc2)cc1</smiles>

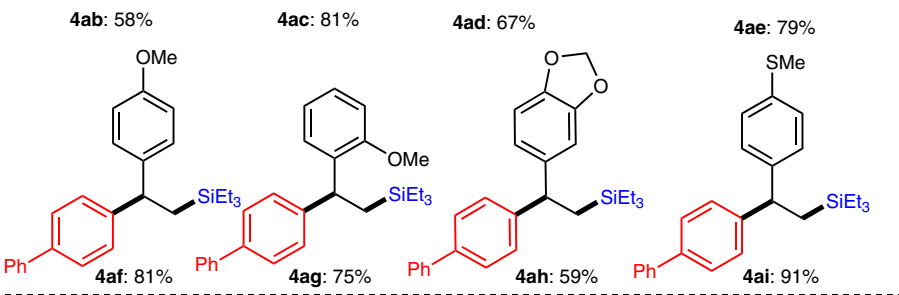

Products with a quaternary carbon center<smiles>CC[Si]CC(CCOC)(c1ccccc1)c1ccc(-c2ccccc2)cc1</smiles>

4aj: $71 \%$

4ak: $90 \%$

4al: $51 \%$<smiles>[14CH3]C(C[Mg])(c1ccccc1)c1ccccc1</smiles><smiles>CCCC(C)(CC)c1ccc(-c2ccc(Cl)cc2)cc1</smiles>

4fk: $73 \%$

$4 \mathrm{mk} \cdot 69 \%$

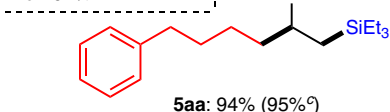

Scope of silyl borates

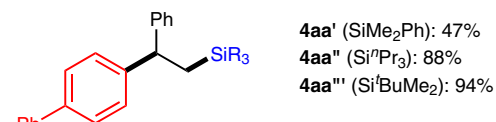
舟

4ea: $90 \%\left(93 \%{ }^{c}\right) \quad$ 4fa: $63 \%^{d}\left(70 \%\right.$,,$\quad$ 4ga: $85 \%^{d}(90 \%, c$, 


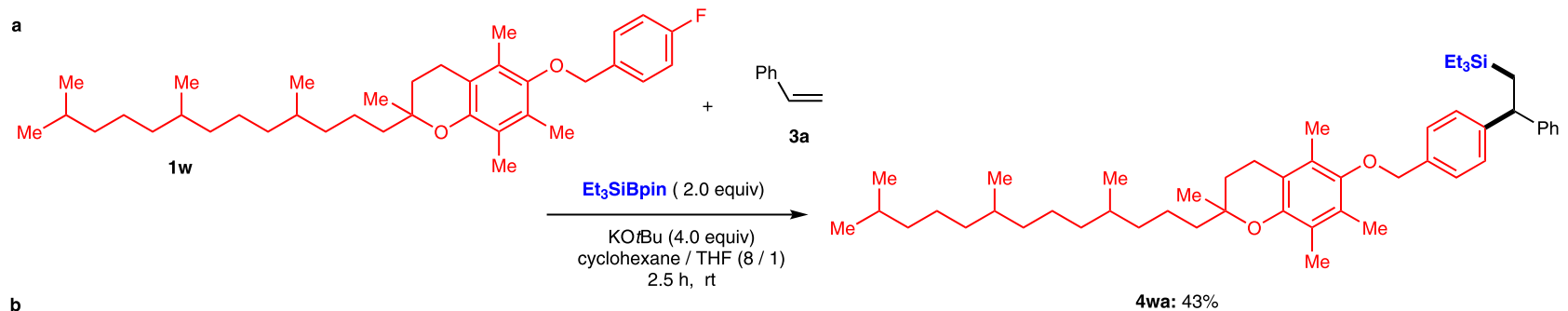

b<smiles>[Y]C(C)[C@H]1CC[C@@H](C)C[C@@H]1OCc1ccc(F)cc1</smiles><smiles>CC[SiH2]C[C@H](c1ccccc1)c1ccc(COC2C[C@H](C)CC[C@H]2C(C)C)cc1</smiles>
$( \pm)-\alpha$-Tocopherol derivative

c<smiles>[CH][C@]12CC[C@@H]3c4ccc(F)cc4CC[C@H]3[C@@H]1CCC2OC</smiles><smiles>C=Cc1ccccc1</smiles>

d<smiles>Fc1ccc(-c2ccccc2)cc1</smiles>

$1 \mathrm{a}$<smiles>C=Cc1ccc2c(c1)CC[C@@H]1[C@@H]2CC[C@@]2(C)[C@H]1CC[C@H]2OC</smiles><smiles>[Y]C[C@H]1CC[C@H](c2ccc(F)cc2)CC1</smiles><smiles>C=Cc1ccc(C#N)cc1</smiles>

3i

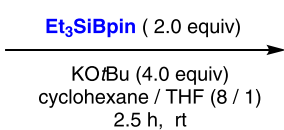

$2.5 \mathrm{~h}, \mathrm{rt}$

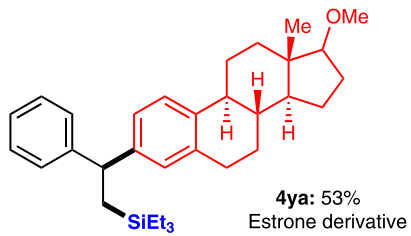

4xa: $55 \%$ Menthol derivative

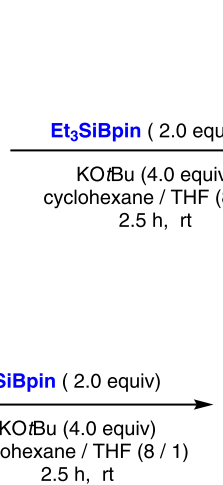
$2.5 \mathrm{~h}$, it

a ( \pm$)-\alpha$-Tocopherol derivative.

b Menthol derivative. c,<smiles>COC1CC[C@H]2[C@H]3CCc4cc([C@@H](C[AsH2])c5ccc(-c6ccccc6)cc5)ccc4[C@]3(C)CC[C@]12C</smiles>
4am: $79 \%$
Estrone derivative

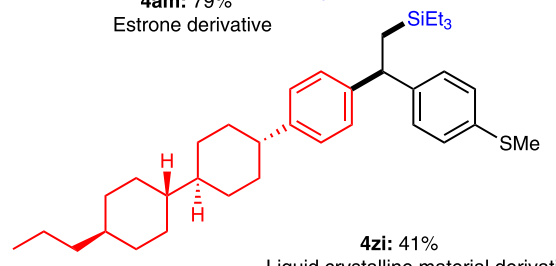

Fig. 3 Substrates relevant to medicinal or materials chemistry. a $( \pm)-\alpha-$ Tocopherol crystalline material derivative.

(3f, 3g), dioxole (3h), and MeS (3i) groups were all, even the highly sterically hindered ortho-MeO-styrene $3 \mathrm{~g}$, well-tolerated as substrates and furnished the desired products $\mathbf{4}$ in good to excellent yield (4ac: 81\%; 4ad: 67\%; 4ae: 79\%; 4af: 81\%; 4ag: 75\%; 4ah: 59\%, 4ai: $91 \%)$. It is important to note that this threecomponent coupling reaction tolerated any combination of the coupling components. A wide variety of substituted or nonsubstituted fluoroarenes 1, aryl alkenes $\mathbf{3}$, and silyl boronates $\mathrm{R}_{3} \mathrm{SiBpin}$ furnished the desired silylated alkanes 4 in good to high yield (4cg: 46\%; 4ci: 33\%; 4lc: 70\%; 4mc: 84\%; 4vi: 65\%; 4ab': $92 \%)$. We also investigated the reaction of substituted styrene derivatives with a quaternary carbon center at the $\beta$-position of the silyl moiety. For example, a, $\alpha$-diphenylethylene $3 \mathbf{j}$, $\alpha$ methylstyrene 3k, and (4-methoxybut-1-en-2-yl)benzene $\mathbf{3 l}$ reacted with fluoroarenes 1 under the standard reaction conditions to give the corresponding products 4 (4aj: 71\%; 4ak: 90\%; 4al: 51\%; 4fk: 73\%; 4mk: 69\%). Furthermore, we attempted the defluorinative alkylsilylation of alkenes using alkyl fluorides with an inert $\mathrm{C}\left(s p^{3}\right)-\mathrm{F}$ bond under the same reaction conditions. Primary alkyl fluorides $\mathbf{2 a}-\mathbf{2 h}$ efficiently reacted regioselectively with styrenes $(\mathbf{3 a}, \mathbf{3 c}, \mathbf{3 i})$ to afford the corresponding alkylsilylation products in up to $94 \%$ yield (5aa: $94 \%$; 5ba: $89 \%$; 5ca: $85 \%$; 5da: $91 \%$; 5ei: $76 \%$; 5fi: $86 \%$; 5gi; $40 \%$; 5hc: $83 \%)$. Notably, the trifluoromethoxy $\left(\mathrm{CF}_{3} \mathrm{O}\right)$ moiety of $\mathbf{2} \mathbf{d}$ remained intact to provide $\mathbf{5 d a}$. Secondary alkyl fluorides $\mathbf{2} \mathbf{i}$ and $\mathbf{2 j}$ also furnished the desired defluorinative alkylsilylation products $\mathbf{5 i a}$ and $\mathbf{5 j a}$ in $36 \%$ and $52 \%$ yield, respectively. The reaction using alkyl fluorides was also carried out in the presence of $\mathrm{Ni}(\mathrm{COD})_{2}$ catalyst $(1 \mathrm{~mol} \%)$ under the best conditions (entry 6, Table 1 ). We obtained almost the same results (72-95\%) as the yields without Ni catalyst. Thus, the defluorinative carbosilylation does not require $\mathrm{Ni}$ catalysis independent of the case of aryl or alkyl fluorides.

To highlight the synthetic utility of this three-component defluorinative carbosilylation reaction, we examined the functionalization of several drug derivatives with fluoroarene moieties (Fig. 3). ( \pm )- $\alpha$-Tocopherol derivative $1 \mathbf{w}$ successfully underwent carbosilylation to afford ( \pm )- $\alpha$-tocopherol derivative 4 wa in $43 \%$ yield (Fig. 3a). (-)-Menthol-derived fluorobenzene $\mathbf{1 x}$ proceeded well under identical conditions to give carbosilylation product 4xa in 55\% yield (Fig. 3b). Steroid derivatives 4 ya and $4 \mathbf{a m}$ were synthesized in $53 \%$ and $79 \%$ yield via (1) the defluorination of fluoro-incorporated estrone derivative $\mathbf{1 y}$ or (2) the defluorination of 1a with alkene- incorporated estrone derivative $3 \mathrm{~m}$ (Fig. 3c, d). Moreover, the liquid crystalline material $\mathbf{1 z}$ was also successfully functionalized using this transformation with $3 \mathbf{i}$ to give $\mathbf{4 z i}$ in $41 \%$ yield (Fig. $3 e$ ).

Furthermore, we examined both the chemoselectivity and siteselectivity of the alkenyl moiety (Fig. 4). (E)-Buta-1,3-dien-1- 


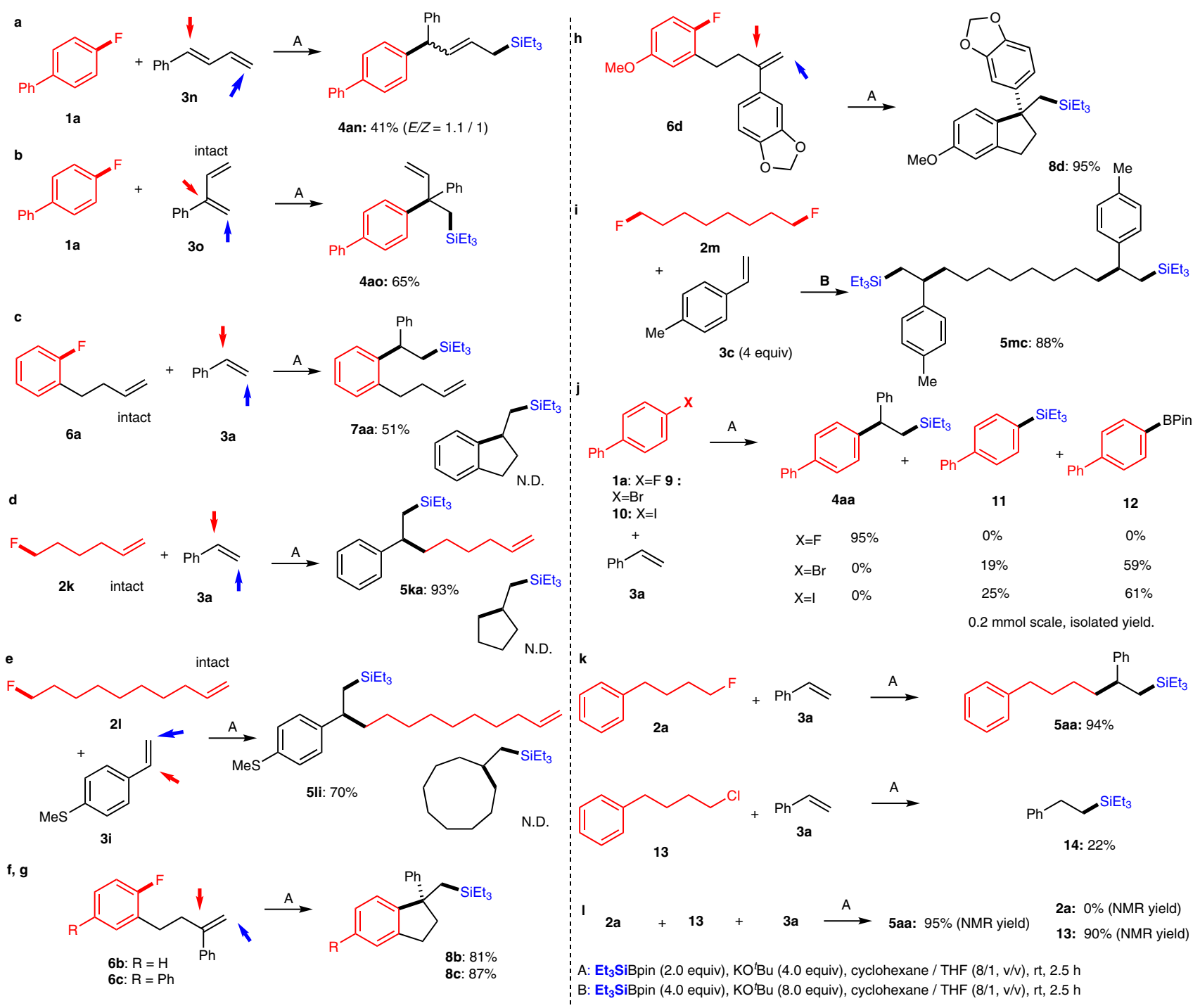

Fig. 4 Chemo- and site-selective coupling reactions. a Selective 1,4-type addition carbosilylation of 1,3-diene 3n. b Selective 1,2-carbosilylation of 1,3-diene 30. $\mathbf{c}-\mathbf{e}$ Selective three-component carbosilylation of styrenes (3a or $\mathbf{3 i}$ ) in the presence of an aliphatic alkene moiety in aryl fluoride $\mathbf{6 a}$ or alkyl fluorides $\mathbf{2 k}$, or $\mathbf{2} \mathbf{l}$. $\mathbf{f - h}$ Intramolecular carbosilylation of $\mathbf{6 b}$-d to provide cyclized silylated products $\mathbf{8} \mathbf{b}-\mathbf{d}$. $\mathbf{i}$ Five-component carbosilylation of styrene $\mathbf{3 c}$ with 1,8difluorooctane ( $\mathbf{2} \mathbf{m})$. j Comparisons of aryl halides (1a, $\mathbf{9}$, and $\mathbf{1 0})$ for the carbosilylation. $\mathbf{k}$ Comparisons of alkyl halides (2a and $\mathbf{1 3})$ for the carbosilylation. I The competitive reaction between $\mathbf{2 a}$ and $\mathbf{1 3}$.

ylbenzene (3n) was site-selectively converted into the 1,4-type arylsilylation product 4 an in $41 \%$ yield with an $E / Z$ ratio of $1.1 / 1$ (determined by ${ }^{1} \mathrm{H}$ nuclear magnetic resonance (NMR) analysis), whereas no 1,2-adduct was observed (Fig. 4a). Conversely, phenoprene 30 preferably furnished 4ao (65\%), a compound with a quaternary carbon center, under the standard reaction conditions via the 1,2-arylsilylation process and not the 1,4process (Fig. 4b). When we examined the reaction of 1-(but-3-en1-yl)-2-fluorobenzene (6a) with $\mathbf{3 a}$ in the presence of $\mathrm{Et}_{3} \mathrm{SiBpin}$, only the three-component condensation product 7 aa was obtained in $51 \%$ yield, whereas the intramolecular product was not detected (Fig. 4c). For the reaction of 6-fluorohex-1-ene (2k) and 10-fluorodec-1-ene (2l) having a terminal olefin moiety with styrenes $\mathbf{3 a}$ and $\mathbf{3} \mathbf{i}$, the three-component condensation products 5ka and 5li were obtained in $93 \%$ and $70 \%$ yields, respectively, whereas the intramolecular products were not detected (Fig. 4d, e). In contrast, an intramolecular carbosilylation was achieved via the reaction of styrene-substituted fluoroarene $\mathbf{6 b}-\mathbf{6} \mathbf{d}$ with $\mathrm{Et}_{3} \mathrm{SiBpin}$ under identical conditions to furnish $\mathbf{8 b}-\mathbf{8 d}$, molecules with a quaternary carbon center, in $81-95 \%$ yield (Fig. $4 \mathrm{f}-\mathrm{h}$ ). The five- component condensation was observed for the reaction of difunctionalized 1,8-difluorooctane (2m) with styrene $3 \mathbf{c}$ and $\mathrm{Et}_{3} \mathrm{SiBpin}$ to provide $5 \mathrm{mc}$ in $88 \%$ yield (Fig. $4 \mathrm{i}$ ). It should be noted that, under the applied conditions, bromo-, and iodosubstituted arenes 9, 10 afforded a mixture of two-component condensation products of silylated 11 and borylated 12 compounds, whereas the targeted three two-component product 4aa was not detected (Fig. 4j). We also attempted the reaction of alkyl chloride $\mathbf{1 3}$ instead of alkyl fluoride $\mathbf{3 a}$ under the same conditions. Although the three-component product 5aa was obtained by alkyl fluoride 3a, the two-component product, triethyl(phenethyl)silane (14), made from $3 \mathbf{a}$ and $\mathrm{Et}_{3} \mathrm{SiBPin}$, was obtained in $22 \%$ yield without incorporation of alkyl chloride 13 (Fig. 4k). Alkyl bromide and iodide were also not converted into 5aa under identical conditions (Supplementary Fig. 5). Encouraged by the results, we attempted the chemoselective activation of the alkyl $\mathrm{C}-\mathrm{F}$ bond over the alkyl $\mathrm{C}-\mathrm{Cl}$ bond by the competitive reaction between $\mathbf{2 a}$ and 13. Interestingly, the alkyl fluoride $\mathbf{2 a}$ was consumed to $5 \mathbf{a a}$ (95\%), whereas the alkyl chloride 13 was recovered (90\%, Fig. 41, more details in Supplementary Figs. 6-8). These behaviors show 

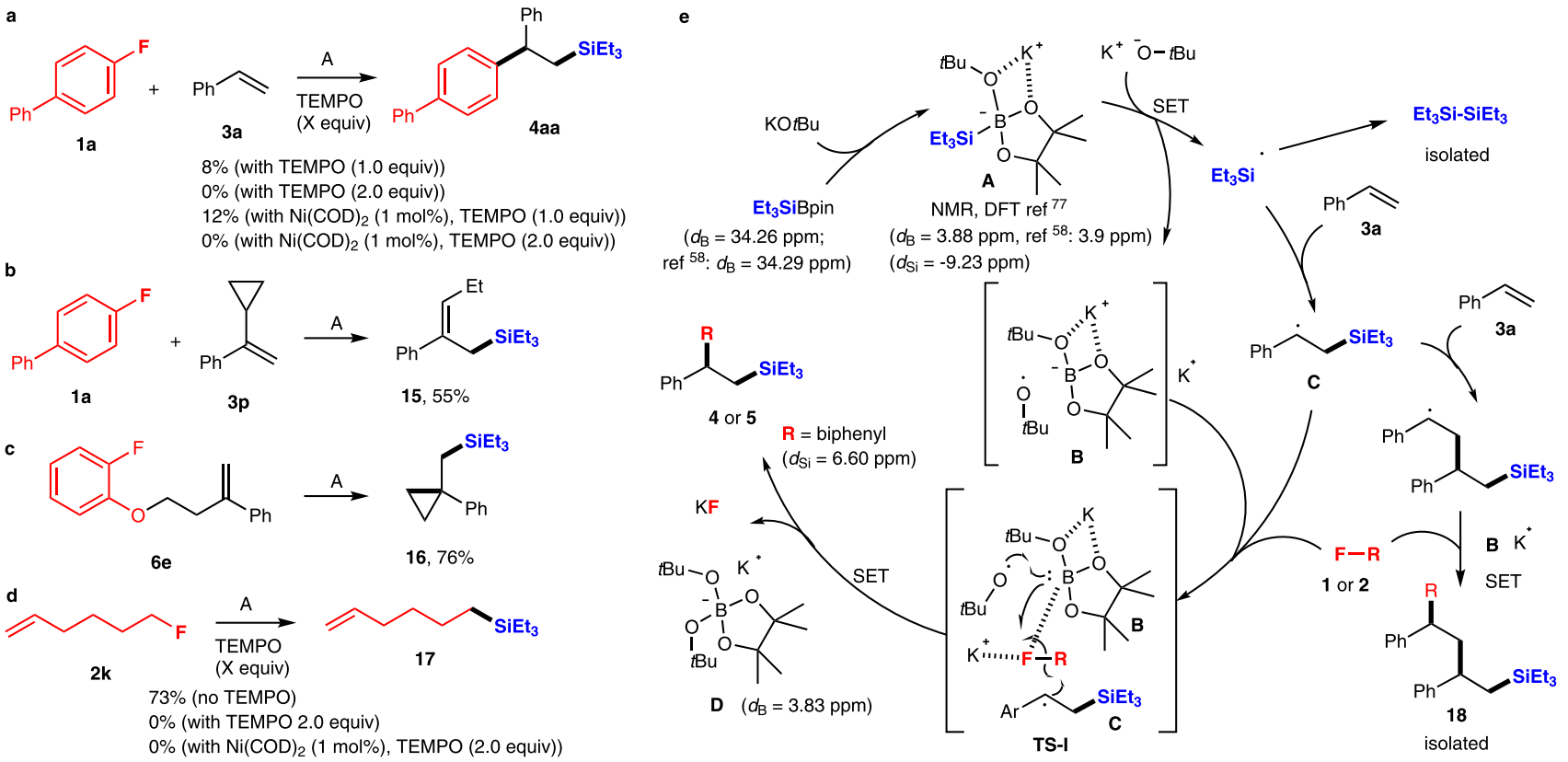<smiles>Fc1ccc(-c2ccccc2)cc1</smiles>

$1 a$

c

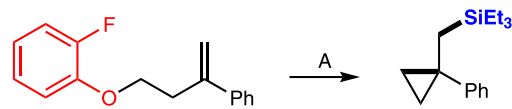

$6 e$
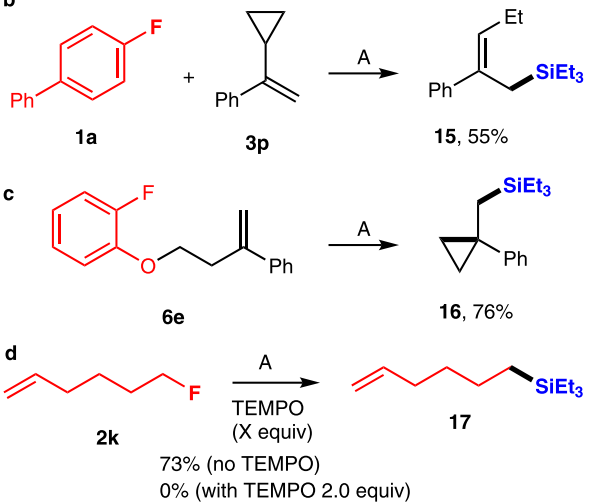

$15,55 \%$

$0 \%$ (with TEMPO 2.0 equiv)
$0 \%$ (with $\mathrm{Ni}(\mathrm{COD})_{2}$ ( 1 mol\%), TEMPO (2.0 equiv))
A: $\mathrm{Et}_{3} \mathrm{SiBpin}$ (2.0 equiv), KO'Bu (4.0 equiv), cyclohexane / THF (8/1, v/v), rt, $2.5 \mathrm{~h}$

Fig. 5 Mechanistic study. a Effect of TEMPO for carbosilylation of $\mathbf{1 a}, \mathbf{3} \mathbf{a}$, and Et ${ }_{3}$ SiBPin. $\mathbf{b}$, c Radical clock experiments. $\mathbf{d}$ Effect of TEMPO for the reaction of alkyl fluoride $\mathbf{2 k}$ and $\mathrm{Et}_{3} \mathrm{SiBPin}$. e Proposed reaction mechanisms.

the advantage of fluorine in our reaction system compared to commonly used halogens both in aromatic and aliphatic cases.

Mechanistic study. Based on this consideration and on the results obtained so far, a radical pathway seems to be a viable hypothesis. To gain an insight into the reaction mechanism, some experiments were undertaken. First the transformation of 1a with $\mathbf{3 a}$ to 4aa under the best conditions was significantly inhibited by the addition of (2,2,6,6-tetramethylpiperidin-1-yl)oxyl (TEMPO) (Fig. 5a). These results strongly suggested the reaction involves radical species. We also attempted the same reaction in the presence of $\mathrm{Ni}$ catalyst. The same results were obtained. We next examined the radical clock experiment with the substrate containing a cyclopropyl moiety at the a-position of the styrene derivative $\mathbf{3 p}$ (Fig. 5b). The silylated ring-opening product 15 was obtained as a major product in 55\% yield and most of the aryl fluoride la was recovered. On the other hand, the expected threecomponent product was not clearly observed, the trace of the desired material was detected only by gas chromatography-mass spectrometry (GCMS). The results are also in good agreement with the radical-mediated reaction mechanism, as the potential cyclopropyl carbinyl radical is known to spontaneously rearrange to allylcarbinyl radical. Furthermore, the substrate $6 \mathrm{e}$ having both fluoroarene and styrene moieties was transformed into the silylcyclopropyl compound $\mathbf{1 6}$ in $76 \%$ yield (Fig. 5c), which also supports the radical pathway. The treatment of $2 \mathbf{k}$ with $\mathrm{Et}_{3} \mathrm{SiBPin}$ in the absence of styrenes $\mathbf{3}$ predominantly gave a silylalkene $\mathbf{1 7}$ in $73 \%$ yield via $\mathrm{C}-\mathrm{F}$ bond activation, whereas the transformation was completely inhibited by the addition of TEMPO independent of the existence of $\mathrm{Ni}$ catalyst (Fig. 5d). Thus, the generation of radical species does not require the reaction with styrenes 3 .

Based on both the experiments' results here and in previous reports ${ }^{44,67-74}$, we propose a single-electron transfer/radicalmediated carbosilylation reaction mechanism triggered by the known ability of $\mathrm{KO} t \mathrm{Bu}$ to serve as a single-electron reductant ${ }^{75,76}$ (Fig. 5e). First, $\mathrm{Et}_{3} \mathrm{SiBpin}$ reacts with a molecule of $\mathrm{KO} \mathrm{tBu}$ to form an intermediate $\mathbf{A}$. The formation of $\mathbf{A}$ has previously been confirmed by the Avasare group based on density functional theory calculations ${ }^{77}$. We also confirmed the intermediate $\mathrm{A}$ by the ${ }^{11} \mathrm{~B}$ NMR and ${ }^{29}$ Si-NMR study (Supplementary Figs. 12-15) ${ }^{58}$. Next, a single-electron transfer process would start by the additional amount of $\mathrm{KO} t \mathrm{Bu}$ as a trigger ${ }^{75,76}$. Namely, a single electron is transferred from $t$-butoxy anion $(-\mathrm{O} t \mathrm{Bu})$ to the silicon atom of intermediate $\mathbf{A}$ to furnish a triethylsilyl radical $\mathrm{SiEt}_{3}$ via a cleavage of the $\mathrm{Si}-\mathrm{B}$ bond. The $t$-butoxy radical ${ }^{\circ} \mathrm{O} t \mathrm{Bu}$ is also generated, which would be captured by the borate anion $\mathbf{B}$. The mechanisms for the cleavage of $\mathrm{Si}-\mathrm{B}$ bonds are various; the radical-mediated Si-B bond cleavage ${ }^{78-80}$ should be highly acceptable due to the experimental results using ${ }^{t} \mathrm{BuOK}^{75,76}$. The generated triethylsilyl radical $\mathrm{SiEt}_{3}$ reacts with styrene $\mathbf{3 a}$ to give a radical adduct $\mathbf{C}$. The radical cascade process should happen between the radical species $\mathrm{C}$, aryl $\mathbf{1}$ or alkyl fluorides $\mathbf{2 , t} t$-butoxy radical ${ }^{\circ} \mathrm{O} t \mathrm{Bu}$, and borate anion $\mathbf{B}$ in the transition state TS-I, where the C-F bond of aryl $\mathbf{1}$ or alkyl fluorides $\mathbf{2}$ is activated by the approach of $\mathrm{K}^{+}$. The boron atom in $\mathbf{B}$ would also participate in activation of the $\mathrm{C}-\mathrm{F}$ bond. Finally, the $\mathrm{C}-\mathrm{C}$ bond formation is completed under concomitant generation of stable D $\left[\mathrm{BPin}(\mathrm{O} t \mathrm{Bu})_{2}\right] \mathrm{K}$ (Supplementary Fig. 14) ${ }^{58}$ and $\mathrm{KF}$ to furnish the desired three-component adduct 4 or $\mathbf{5}$. The generation of benzyl radical species $\mathbf{C}$ was supported by the cyclopropyl experiments (Fig. 5b, c). It should be noted that through the experiments, we observed side products such as $\mathrm{Et}_{3} \mathrm{Si}$ $\mathrm{SiEt}_{3}$ and double styrene adducts 18. These formations can be explained by the dimerization of triethylsilyl radical ${ }^{\prime} \mathrm{SiEt}_{3}$ and the overreaction of benzyl radical $\mathbf{C}$ with styrene $\mathbf{3 a}$.

In conclusion, we have developed a carbosilylation of alkenes that uses silyl boronates and organic fluorides, and that proceeds via the activation of an inert $\mathrm{C}-\mathrm{F}$ bond without a catalyst. This reaction should be initiated by the radical cleavage of $\mathrm{Si}-\mathrm{B}$ bond via a single-electron transfer from ${ }^{t} \mathrm{BuOK}$. A variety of $\beta$ functionalized silyl compounds can be synthesized efficiently and rapidly in good to excellent yield under very mild conditions at room temperature. The most significant feature of this protocol is its broad substrate scope. This highly efficient protocol accepts a variety of fluorides, including aryl and alkyl fluorides, and even 
transforms sterically demanding secondary alkyl fluorides. A broad range of aryl alkenes, such as styrene derivatives and $\alpha$ substituted aryl alkenes, are also tolerated by this method. Silyl boronates were identified as viable substrates. Moreover, the chemo- and site-selectivity displayed in this reaction are remarkable. Aryl alkenes selectively react in the presence of non-aryl alkenes and an intramolecular carbosilylation was achieved with substrates that possess both fluoroarene and aryl alkene moieties. The tolerance of the reaction toward different functional groups is also significant. Substrates with ether, $\mathrm{CF}_{3}$, and hetero-aromatic moieties react smoothly without the detection of $\mathrm{C}-\mathrm{O}$ cleavage, $\mathrm{C}-\mathrm{F}$ bond activation, or $\mathrm{C}-\mathrm{H}$ activation; only the inert $\mathrm{C}-\mathrm{F}$ bonds of the fluoroarenes and fluoroalkanes are activated. Notably, this method also allows for the synthesis of silyl compounds with a quaternary carbon center at the $\beta$-position. The 1,4-type addition carbosilylation of 1,3dienes was also achieved. Given the high number of fluorinated compounds that are commercially available, including structurally complex pharmaceuticals, agrochemicals, and functional materials, this protocol widens the potential utility of organosilicon compounds in organic synthesis, the structural design of lead drug compounds, and functional materials.

Finally, the limitations of the method should be mentioned. The conjugated diene is acceptable but electron-deficient acrylates and acrylamides are not suitable. Internal styrenes such as cisstilbene and trans- $\beta$-methylstyrene did not react (Supplementary Figs. 1 and 2). The chemoselective activation of aromatic $\mathrm{C}-\mathrm{F}$ bonds over aromatic $\mathrm{C}-\mathrm{Br}$ and $\mathrm{C}-\mathrm{I}$ bonds is difficult, whereas the only aromatic fluoride was transformed into the desired threecomponent product (Supplementary Figs. 3 and 4).

\section{Methods}

General procedure for the defluorinative carbosilylation of alkenes 3 using $\mathbf{R}_{\mathbf{3}} \mathrm{SiBpin}$ and aryl fluorides $\mathbf{1}$ or alkyl fluorides $\mathbf{2}$. In a $\mathrm{N}_{2}$-filled glovebox to a flame-dried screw-capped test tube were added organic fluorides 1 or $\mathbf{2}(0.20 \mathrm{mmol}$, 1.0 equiv), silyl boronates $\mathrm{R}_{3} \mathrm{SiBpin}(0.4 \mathrm{mmol}, 2.0$ equiv), alkenes $3(0.40 \mathrm{mmol}$, 2.0 equiv), $\mathrm{KO} t \mathrm{Bu}(90 \mathrm{mg}, 0.8 \mathrm{mmol}, 4.0$ equiv), and cyclohexane $/ \mathrm{THF}(1.5 \mathrm{~mL}, 8 /$ $1, v / v)$ sequentially. The tube was then sealed and removed from the glovebox. The mixture was stirred at room temperature for $2.5 \mathrm{~h}$. To the reaction tube was added hexane $(5 \mathrm{~mL})$ and then it was subjected to filter through a short silica pad, washed with $\mathrm{Et}_{2} \mathrm{O}$, and concentrated under vacuum, followed by 3 -fluoropyridine $(8.6 \mu \mathrm{L}$, $0.1 \mathrm{mmol}$ ) as an internal standard for NMR analysis. The mixture was then concentrated again to give the residue, which was purified by column chromatography on silica gel to give the corresponding carbosilylation products $\mathbf{4}$ or $\mathbf{5}$.

\section{Data availability}

The data supporting the findings of this study are available within the paper and its Supplementary Information. All relevant data are also available from the authors.

Received: 16 December 2020; Accepted: 1 June 2021; Published online: 18 June 2021

\section{References}

1. Dhungana, R. K. \& Giri, R. Transition metal-catalyzed dicarbofunctionalization of unactivated olefins. Chem. Rec. 18, 1314-1340 (2018).

2. Derosa, J. et al. Recent developments in nickel-catalyzed intermolecular dicarbofunctionalization of alkenes. Chem. Sci. 11, 4287-4296 (2020).

3. Yin, G. Y., Mu, X. \& Liu, G. S. Palladium(II)-catalyzed oxidative difunctionalization of alkenes: bond forming at a high-valent palladium center. Acc. Chem. Res. 49, 2413-2423 (2016).

4. McDonald, R. I., Liu, G. \& Stahl, S. S. Palladium(II)-catalyzed alkene functionalization via nucleopalladation: stereochemical pathways and enantioselective catalytic applications. Chem. Rev. 111, 2981-3019 (2020).

5. Li, Z. L. et al. Recent advances in copper-catalysed radical-involved asymmetric 1,2-difunctionalization of alkenes. Chem. Soc. Rev. 49, 32-48 (2020).

6. Jiang, H. \& Studer, A. Intermolecular radical carboamination of alkenes. Chem. Soc. Rev. 49, 1790-1811 (2020).
7. Tu, H. Y. et al. Recent advances in nickel-catalyzed three-component difunctionalization of unactivated alkenes. Synthesis 52, 1346-1356 (2020).

8. Semba, K. \& Nakao, Y. Arylboration of alkenes by cooperative palladium/ copper catalysis. J. Am. Chem. Soc. 136, 7567-7570 (2014).

9. Logan, K. M. et al. Nickel-catalyzed stereoselective arylboration of unactivated alkenes. J. Am. Chem. Soc. 140, 159-162 (2018).

10. Chen, L. A. et al. Nickel-catalyzed arylboration of alkenylarenes: synthesis of boron substituted quaternary carbons and regiodivergent reactions. Angew. Chem. Int. Ed. 58, 1-6 (2019).

11. Huang, H. M. et al. Three-component, interrupted radical Heck/allylic substitution cascade involving unactivated alkyl bromides. J. Am. Chem. Soc. 142, 10173-10183 (2020).

12. Derosa, J. et al. Nickel-catalyzed 1,2-diarylation of simple alkenyl amides. J. Am. Chem. Soc. 140, 17878-17883 (2018).

13. García-Domínguez, A., Li, Z. \& Nevado, C. Nickel-catalyzed reductive dicarbofunctionalization of alkenes. J. Am. Chem. Soc. 139, 6835-6838 (2017).

14. Rosen, B. R., Ney, J. E. \& Wolfe, J. P. Use of aryl chlorides as electrophiles in Pd-catalyzed alkene difunctionalization reactions. J. Org. Chem. 75, 2756-2759 (2010).

15. Lan, Y. \& Wang, C. Nickel-catalyzed enantioselective reductive carboacylation of alkenes. Commun. Chem. 3, 45 (2020).

16. Yang, X. et al. Advances in catalytic enantioselective fluorination, mono-, di-, and trifluoromethylation, and trifluoromethylthiolation reactions. Chem. Rev. 115, 826-870 (2015).

17. Champagne, P. A. et al. Monofluorination of organic compounds: 10 years of innovation. Chem. Rev. 115, 9073-9174 (2015).

18. Zhu, Y. et al. Modern approaches for asymmetric construction of carbon-fluorine quaternary stereogenic centers: synthetic challenges and pharmaceutical needs. Chem. Rev. 118, 3887-3964 (2018).

19. Inoue, M., Sumii, Y. \& Shibata, N. Contribution of organofluorine compounds to pharmaceuticals. ACS Omega 5, 10633-10640 (2020).

20. Ogawa, Y. et al. Current contributions of organofluorine compounds to the agrochemical industry. iScience 23, 101467 (2020).

21. Ahrens, T. et al. Functionalization of fluorinated molecules by transitionmetal-mediated C-F bond activation to access fluorinated building blocks. Chem. Rev. 115, 931-972 (2015).

22. Eisenstein, O., Milani, J. \& Perutz, R. N. Selectivity of C-H activation and competition between $\mathrm{C}-\mathrm{H}$ and $\mathrm{C}-\mathrm{F}$ bond activation at fluorocarbons. Chem. Rev. 117, 8710-8753 (2017).

23. Hamel, J. D. \& Paquin, J. F. Activation of C-F bonds a to C-C multiple bonds. Chem. Commun. 54, 10224-10239 (2018).

24. Kuehnel, M. F., Lentz, D. \& Braun, T. Synthesis of fluorinated building blocks by transition-metal-mediated hydrodefluorination reactions. Angew. Chem. Int. Ed. 52, 3328-3348 (2013).

25. Tobisu, M. et al. Nickel-catalyzed Suzuki-Miyaura reaction of aryl fluorides. J. Am. Chem. Soc. 133, 19505-19511 (2011).

26. Guo, W. H. et al. Rhodium-catalyzed ortho-selective C-F bond borylation of polyfluoroarenes with Bpin-Bpin. Angew. Chem. Int. Ed. 54, 9075-9078 (2015).

27. Niwa, T. et al. Ni/Cu-catalyzed defluoroborylation of fluoroarenes for diverse C-F bond functionalizations. J. Am. Chem. Soc. 137, 14313-14318 (2015).

28. Liu, X. W. et al. Ni-catalyzed borylation of aryl fluorides via C-F cleavage. J. Am. Chem. Soc. 137, 12470-12473 (2015).

29. O’Neill, M. J., Riesebeck, T. \& Cornella, J. Thorpe-ingold effect in branchselective alkylation of unactivated aryl fluorides. Angew. Chem. Int. Ed. 57, 9103-9107 (2018).

30. Liu, X. W., Zarate, C. \& Martin, R. Base-mediated defluorosilylation of C (sp2)-F and C(sp3)-F bonds. Angew. Chem. Int. Ed. 58, 2064-2068 (2019).

31. Stahl, T., Klare, H. F. T. \& Oestreich, M. Main-group Lewis acids for C-F bond activation. ACS Catal. 3, 1578-1587 (2013).

32. Shen, Q. et al. Review of recent advances in C-F bond activation of aliphatic fluorides. J. Fluor. Chem. 179, 14-22 (2015).

33. Iwamoto, $\mathrm{H}$. et al. Cleavage of $\mathrm{C}(\mathrm{sp} 3)-\mathrm{F}$ bonds in trifluoromethylarenes using a bis(NHC)nickel(0) complex. J. Am. Chem. Soc. 142, 19360-19367 (2020).

34. Haruo, S. \& Wataru, A. Palladium catalyzed reactions of neopentylidenesiliranes. Chem. Lett. 17, 1567-1570 (1988)

35. Wu, M. Y., Yang, F. Y. \& Cheng, C. H. Carbosilylation of allenes catalyzed by palladium complexes: a new efficient route to substituted allylic silanes. J. Org. Chem. 64, 2471-2474 (1999).

36. Yeon, S. H. et al. Aluminum chloride catalyzed regioselective allylsilylation of alkenes: convenient route to 5-silyl-1-alkenes. Organometallics 14, 361-2365 (1995).

37. Nii, S., Terao, J. \& Kambe, N. Titanocene-catalyzed carbosilylation of alkenes and dienes using alkyl halides and chlorosilanes. J. Org. Chem. 65, 5291-5297 (2000).

38. Tani, Y. et al. Copper-catalyzed regiodivergent silacarboxylation of allenes with carbon dioxide and a silylborane. J. Am. Chem. Soc. 136, 17706-17709 (2014). 
39. Welle, A. et al. Copper-catalysed domino silylative aldol reaction leading to stereocontrolled chiral quaternary carbons. Chem. Eur. J. 16, 10980-10983 (2010).

40. Obora, Y., Tsuji, Y. \& Kawamura, T. Palladium-catalyzed decarbonylative coupling of acid chlorides, organodisilanes, and 1,3-dienes. J. Am. Chem. Soc. 115, 10414-10415 (1993).

41. Obora, Y., Tsuji, Y. \& Kawamura, T. 1,4-Carbosilylation of 1,3-dienes via palladium catalyzed three-component coupling reaction. J. Am. Chem. Soc. 117, 9814-9821 (1995).

42. Liu, Z. et al. Palladium(0)-catalyzed directed syn-1,2-carboboration and -silylation: alkene scope, applications in dearomatization, and stereocontrol by a chiral auxiliary. Angew. Chem. Int. Ed. 58, 17068-17073 (2019).

43. Peng, H. et al. Radical 1,2-aryl migration in $a, a$-diaryl allylic alcohols toward $\beta$-silyl ketones. Org. Biomol. Chem. 13, 10299-10302 (2015).

44. Zhang, L., Liu, D. \& Liu, Z. Q. A free radical cascade silylarylation of activated alkenes: highly selective activation of the $\mathrm{Si}-\mathrm{H} / \mathrm{C}-\mathrm{H}$ bonds. Org. Lett. 17, 2534-2537 (2015).

45. Yang, Y. et al. Iron-catalyzed intermolecular 1,2-difunctionalization of styrenes and conjugated alkenes with silanes and nucleophiles. Angew. Chem. Int. Ed. 56, 7916-7919 (2017).

46. Hou, J. et al. Visible-light-mediated metal-free difunctionalization of alkenes with $\mathrm{CO}_{2}$ and silanes or $\mathrm{C}(\mathrm{sp} 3)-\mathrm{H}$ alkanes. Angew. Chem. Int. Ed. 57, 17220-17224 (2018).

47. Yang, Y. et al. Oxidative radical divergent Si-incorporation: facile access to Sicontaining heterocycles. Chem. Commun. 54, 1441-1444 (2018).

48. Zhang, H. et al. Copper-catalyzed heteroarylsilylation of unactivated olefins through distal heteroaryl migration. Chem. Asian J. 13, 2453-2457 (2018).

49. Zhang, Z. \& Hu, X. L. Arylsilylation of electron-deficient alkenes via cooperative photoredox and nickel catalysis. ACS Catal. 10, 777-782 (2020).

50. Denmark, S. E. \& Sweis, R. F. Design and implementation of new, siliconbased, cross-coupling reactions: importance of silicon-oxygen bonds. Acc. Chem. Res. 35, 835-846 (2002).

51. Nakao, Y. \& Hiyama, T. Silicon-based cross-coupling reaction: an environmentally benign version. Chem. Soc. Rev. 40, 4893-4901 (2011).

52. Sun, D. et al. Arylsilanes and siloxanes as optoelectronic materials for organic light-emitting diodes (OLEDs). J. Mater. Chem. C. 3, 9496-9508 (2015).

53. Liu, X. M. et al. Highly efficient blue-light-emitting glass-forming molecules based on tetraarylmethane/silane and fluorene: synthesis and thermal, optical, and electrochemical properties. Chem. Mater. 17, 434-441 (2005).

54. Ponomarenko, S. A. \& Kirchmeyer, S. Conjugated organosilicon materials for organic electronics and photonics. Silicon Polym. 235, 33-110 (2010).

55. Franz, A. K. \& Wilson, S. O. Organosilicon molecules with medicina applications. J. Med. Chem. 56, 388-405 (2013).

56. Obligacion, J. V. \& Chirik, P. J. Earth-abundant transition metal catalysts for alkene hydrosilylation and hydroboration. Nat. Rev. Chem. 2, 15-34 (2018).

57. Du, X. Y. \& Huang, Z. Advances in base-metal-catalyzed alkene hydrosilylation. ACS Catal. 7, 1227-1243 (2017)

58. Cui, B. et al. Defluorosilylation of fluoroarenes and fluoroalkanes. Nat. Commun. 9, 4393 (2018)

59. Wang, J., Ogawa, Y. \& Shibata, N. Selective synthesis of spirobiindanes, alkenyl chlorides, and monofluoroalkenes from unactivated gem-difluoroalkanes controlled by aluminum-based Lewis acids. Sci. Rep. 9, 19113 (2019).

60. Wang, J., Ogawa, Y. \& Shibata, N. Activation of saturated fluorocarbons to synthesize spirobiindanes, monofluoroalkenes, and indane derivatives. iScience 17, 132-143 (2019).

61. Tanaka, J. et al. Asymmetric desymmetrization via metal-free $\mathrm{C}-\mathrm{F}$ bond activation: synthesis of 3,5-diaryl-5-fluoromethyloxazolidin-2-ones with quaternary carbon centers. Angew. Chem. Int. Ed. 55, $9432-9436$ (2016)

62. Yu, D. G., Li, B. J. \& Shi, Z. J. Exploration of new C-O electrophiles in crosscoupling reactions. Acc. Chem. Res. 43, 1486-1495 (2010).

63. MacQueen, P. M. et al. Exploiting ancillary ligation to enable nickel-catalyzed C-O cross-couplings of aryl electrophiles with aliphatic alcohols. J. Am. Chem. Soc. 140, 5023-5027 (2018).

64. Toutov, A. A. et al. Silylation of $\mathrm{C}-\mathrm{H}$ bonds in aromatic heterocycles by an earth-abundant metal catalyst. Nature 518, 80-84 (2015).

65. Gu, Y. T. et al. A mild and direct site-selective sp2 C-H silylation of (poly) azines. J. Am. Chem. Soc. 141, 127-132 (2019).

66. Yuan, X. et al. Photocatalytic radical defluoroalkylation of unactivated alkenes via distal heteroaryl ipso-migration. Commun. Chem. 3, 98 (2020).

67. Li, Z. X. et al. Radical addition enables 1,2-aryl migration from a vinylsubstituted all-carbon quaternary center. Angew. Chem. Int. Ed. 60, 186-190 (2021).

68. Jenkins, I. D. \& Krenske, E. H. Mechanistic aspects of hydrosilane/potassium tertbutoxide $\left(\mathrm{HSiR}_{3} / \mathrm{KO} t \mathrm{Bu}\right)$-mediated reactions. ACS Omega 5, 7053-7058 (2020).

69. Alberti, A. \& Pedulli, G. F. Addition reactions of silyl radicals to unsaturated compounds. Rev. Chem. Intermed. 8, 207-246 (1987).
70. Chatgilialoglu, C. Structural and chemical properties of silyl radicals. Chem. Rev. 95, 1229-1251 (1995).

71. Chatgilialoglu, C. et al. Thirty years of (TMS)3SiH: a milestone in radicalbased synthetic chemistry. Chem. Rev. 118, 6516-6572 (2018).

72. Liu, W. B. et al. Potassium tert-butoxide-catalyzed dehydrogenative C-H silylation of heteroaromatics: a combined experimental and computational mechanistic study. J. Am. Chem. Soc. 139, 6867-6879 (2017).

73. Shang, X. \& Liu, Z. Q. Recent developments in free-radical-promoted C-Si formation via elective $\mathrm{C}-\mathrm{H} / \mathrm{Si}-\mathrm{H}$ functionalization. Org. Biomol. Chem. 14, 7829-7831 (2016)

74. Li, J. S. \& Wu, J. Recent developments in the photo-mediated generation of silyl radicals and their application in organic synthesis. ChemPhotoChem 2, 839-846 (2018).

75. Nocera, G. et al. Electron transfer reactions: $\mathrm{KO} t \mathrm{Bu}$ (but not $\mathrm{NaO} t \mathrm{Bu}$ ) photoreduces benzophenone under activation by visible light. J. Am. Chem. Soc. 140, 9751-9757 (2018)

76. Barham, J. P. et al. $\mathrm{KO} t \mathrm{Bu}$ : a privileged reagent for electron transfer reactions? J. Am. Chem. Soc. 138, 7402-7410 (2016).

77. Jain, P., Pal, S. \& Avasare, V. Ni(COD) $)_{2}$-catalyzed ipso-silylation of 2methoxynaphthalene: a density functional theory study. Organometallics 37, 1141-1149 (2018)

78. Oestreich, M., Hartmann, E. \& Mewald, M. Activation of the Si-B interelement bond: mechanism, catalysis, and synthesis. Chem. Rev. 113, 402-441 (2013)

79. Moon, J., Baek, H. \& Kim, J. Mechanistic investigation of thermal and photoreactions between boron and silane. J. Phys. Chem. A 121, 6531-6537 (2017).

80. Feng, J. J. et al. Activation of the $\mathrm{Si}-\mathrm{B}$ interelement bond related to catalysis. Chem. Soc. Rev. 50, 2010-2073 (2021).

\section{Acknowledgements}

This work was supported by JSPS KAKENHI grants JP 18H02553 and 21H01933 (KIBAN B, N.S.).

\section{Author contributions}

N.S. conceived the concept. J.Z. optimized the reaction conditions. J.Z., B.J., Y.F., Z.Z, and T.I. surveyed the substrate scope. J.Z., B.J., and Y.F. prepared staring materials. N.S directed the project. N.S. and J.Z. prepared the manuscript.

\section{Competing interests}

The authors declare no competing interests.

\section{Additional information}

Supplementary information The online version contains supplementary material available at https://doi.org/10.1038/s41467-021-24031-w.

Correspondence and requests for materials should be addressed to N.S.

Peer review information Nature Communications thanks the anonymous reviewer(s) for their contribution to the peer review of this work. Peer reviewer reports are available.

Reprints and permission information is available at http://www.nature.com/reprints

Publisher's note Springer Nature remains neutral with regard to jurisdictional claims in published maps and institutional affiliations.

Open Access This article is licensed under a Creative Commons Attribution 4.0 International License, which permits use, sharing, adaptation, distribution and reproduction in any medium or format, as long as you give appropriate credit to the original author(s) and the source, provide a link to the Creative Commons license, and indicate if changes were made. The images or other third party material in this article are included in the article's Creative Commons license, unless indicated otherwise in a credit line to the material. If material is not included in the article's Creative Commons license and your intended use is not permitted by statutory regulation or exceeds the permitted use, you will need to obtain permission directly from the copyright holder. To view a copy of this license, visit http://creativecommons.org/ licenses/by/4.0/.

(C) The Author(s) 2021 